\title{
A Circadian Clock and Light/Dark Adaptation Differentially Regulate Adenosine in the Mammalian Retina
}

\author{
Christophe Ribelayga and Stuart C. Mangel \\ Department of Neurobiology, Civitan International Research Center, University of Alabama School of Medicine, Birmingham, Alabama $35294-0021$
}

\begin{abstract}
Although the purine adenosine acts as an extracellular neuromodulator in the mammalian CNS in both normal and pathological conditions and regulates sleep, the regulation of extracellular adenosine in the day and night is incompletely understood. To determine how extracellular adenosine is regulated, rabbit neural retinas were maintained by superfusion at different times of the regular light/dark and circadian cycles. The adenosine level in the superfusate, representing adenosine overflow from the retinas, and the adenosine level in retinal homogenates, representing adenosine content, were measured using HPLC with fluorescence detection in the absence or presence of blockers of adenosine transport and/or extracellular adenosine synthesis. We report that darkness, compared with illumination, increases the level of extracellular adenosine, and that a circadian clock also increases extracellular adenosine at night. In addition, we show that the darkness-evoked increase in the level of extracellular adenosine results primarily from an increase in the conversion of extracellular ATP into adenosine, but that the clock-induced increase at night results primarily from an increase in the accumulation of intracellular adenosine. We also show that a slightly hypoxic state increases adenosine content and overflow to an extent similar to that of the clock. Our findings demonstrate that the extracellular level of adenosine in the mammalian retina is differentially regulated by a circadian clock and the lighting conditions and is maximal at night under dark-adapted conditions. We conclude that adenosine is a neuromodulator involved in both circadian clock and dark-adaptive processes in the vertebrate retina.
\end{abstract}

Key words: adenosine; circadian rhythm; circadian clock; retina; dark adaptation; light adaptation

\section{Introduction}

In the CNS, the purine adenosine plays numerous modulatory roles in both normal and pathological conditions including the modulation of neurotransmission, sleeping-waking, blood flow, inflammation, and pain, and the response to hypoxia and ischemia (Dunwiddie and Masino, 2001; Latini and Pedata, 2001). The extracellular level of adenosine increases in response to various stimuli such as increased tissue activity, hypoxia, and stress. The accumulation of extracellular adenosine arises from two different sources, either from the conversion of extracellular ATP into adenosine via the sequential actions of an ectoATPase and an ectonucleotidase or from the intracellular conversion of AMP via the action of an endonucleotidase. In this latter case, if the intracellular concentration of adenosine exceeds that on the outside, as occurs during ischemia-hypoxia, it is transported out. Clearance from the extracellular space requires reuptake and intracellular breakdown of adenosine by adenosine deaminase or adenosine kinase (Dunwiddie and Masino, 2001; Latini and Pedata, 2001).

\footnotetext{
Received May 4, 2004; revised Nov. 11, 2004; accepted Nov. 12, 2004.

This work was supported in part by a grant to S.C.M. from the National Institutes of Health (NIH) (EY005102), by a National Eye Institute Core grant (EY03039) to the University of Alabama at Birmingham, and by an NIH grant (HD38985).C.R. was supported in part by a Postdoctoral Fellowship from Fight for Sight-Prevent Blindness America, New York, New York (PD 01009). We thank Dr. Andrey Dmitriev for helpful discussions.

Correspondence should be addressed to Stuart C. Mangel, Department of Neurobiology, University of Alabama at Birmingham School of Medicine, Civitan International Research Center, Room 425, 1719 Sixth Avenue South, Birmingham, AL 35294-0021. E-mail: mangel@uab.edu.

DOI:10.1523/JNEUROSCI.3138-04.2005

Copyright $\odot 2005$ Society for Neuroscience $\quad 0270-6474 / 05 / 250215-08 \$ 15.00 / 0$
}

Although the circadian $(24 \mathrm{hr})$ clock in the suprachiasmatic nucleus controls sleeping-waking, and although adenosine has been implicated in the regulation of sleep (Porkka-Heiskanen et al., 1997; Shiromani et al., 1998; Pace-Schott and Hobson, 2002), it is not known whether a circadian clock regulates adenosine levels. Because a variety of cellular processes in the vertebrate retina exhibit circadian rhythmicity (Cahill and Besharse, 1995; Barlow, 2001; Mangel, 2001; Green and Besharse, 2004), including neural activity (Wang and Mangel, 1996; Ribelayga et al., 2004), pH (Dmitriev and Mangel, 2000, 2001), and energy metabolism (Dmitriev and Mangel, 2004), and because neural activity, $\mathrm{pH}$, and energy metabolism regulate adenosine in the CNS (Doolette, 1997; Dunwiddie and Masino, 2001), we investigated whether a circadian clock regulates adenosine in the mammalian retina.

In fact, a growing body of evidence indicates that adenosine is an important neuromodulator in the vertebrate retina (Blazynski and Perez, 1991; Paes-De-Carvalho, 2002), as indicated by the presence of retinal adenosine metabolic pathway enzymes and receptor subtypes $A_{1}$ and $A_{2 A}$ and by the effects of exogenous adenosine on the physiology of retinal neurons. However, the sources of extracellular adenosine in the retina are unknown and direct measurements of how light/dark adaptation affects endogenous adenosine are still lacking.

In the present paper, we studied the mechanisms that regulate the extracellular level of endogenous adenosine in the mammalian retina in the day and night. Using HPLC with fluorescence detection to measure adenosine overflow and content, we find 
that light and darkness and a circadian clock modulate the level of extracellular adenosine, so that it is high in the dark compared with the light and especially high in the dark at night. We further establish that darkness and the clock increase the extracellular level of adenosine through different means, namely, by increasing the extracellular synthesis of adenosine and by increasing intracellular adenosine content, respectively. Finally, we show that the clock and a slightly hypoxic state increase adenosine content and overflow to a similar extent, a finding that supports the idea that the clock-induced increase in energy metabolism and oxygen consumption at night produces a slight hypoxia.

\section{Materials and Methods}

Tissue preparation. Experiments were performed on superfused retinas obtained from pigmented Dutch-belted adult rabbits weighing 3.0-4.0 $\mathrm{kg}$. Before an experiment, the rabbits were maintained for at least 2 weeks on a $12 \mathrm{hr}$ light/dark cycle (with lights on at 5:00 A.M.). The care and use of the rabbits were in accordance with all guidelines of the National Institutes of Health, the Society for Neuroscience, and the University of Alabama at Birmingham Institutional Animal Care and Use Committee.

Rabbits were dark adapted for at least $1 \mathrm{hr}$ before all experiments. Rabbits were kept in darkness for 24-48 hr at the start of circadian experiments. They were deeply anesthetized with urethane $(1.5 \mathrm{gm} / \mathrm{kg}$, i.p.), and both eyes were enucleated after additional local intraorbital injections of $2 \%$ xylocaine. Surgery was performed under dim red illumination. The eyeball was opened in the equatorial plane, and cuts were made to flatten the eyecup. The vitreous humor was removed with an ophthalmic sponge, and the neural retina from the superior part of the eyecup was gently peeled away from the pigment epithelium and placed in a custom-made closed superfusion chamber $(1 \mathrm{ml})$ in a warm waterbath to maintain the temperature inside the chamber at $33 \pm 1{ }^{\circ} \mathrm{C}$. A peristaltic pump delivered the superfusion solution at a constant rate of $0.5 \mathrm{ml} / \mathrm{min}$, so that the superfusate inside the chamber was replaced every $2 \mathrm{~min}$. The superfusion solution contained (in $\mathrm{mm}$ ): $117 \mathrm{NaCl}, 3.1 \mathrm{KCl}$, $30 \mathrm{NaHCO}_{3}, 2 \mathrm{CaCl}_{2}, 1.2 \mathrm{MgSO}_{4}-7 \mathrm{H}_{2} \mathrm{O}, 10$ glucose, $0.5 \mathrm{NaH}_{2} \mathrm{PO}_{4}$, and $0.1 \mathrm{~L}$-glutamate. Addition of glutamate to the Ringer's solution maintains the glutamate-glutamine cycle and enhances the viability of the retina. However, we ascertained that glutamate did not interfere with our adenosine measurements. Removal of glutamate during a $30 \mathrm{~min}$ period during the day did not affect adenosine overflow from superfused rabbit retinas $(n=4)$ (data not shown). The $\mathrm{pH}$ of the superfusate was maintained at 7.4 by bubbling with a $5 \% \mathrm{CO}_{2}$ gas mixture. Unless specified, the gas mixture also contained $95 \% \mathrm{O}_{2}$. However, in some experiments, the proportion of $\mathrm{O}_{2}$ was lowered and replaced by $\mathrm{N}_{2}$, but the mixture always contained $5 \% \mathrm{CO}_{2}$. In all the experiments, adenosine was assayed in $0.5 \mathrm{ml}$ of superfusate, which corresponds to $1 \mathrm{~min}$ of superfusion. At the end of each experiment, the retina was homogenized in a solution containing $0.1 \mathrm{~m}$ perchloric acid, $5 \mathrm{~mm}$ EDTA, and $1 \mathrm{~mm} \mathrm{Na}^{+}$metabisulfite. A fraction of the homogenate $(100 \mu \mathrm{l})$ was processed to determine adenosine content. The remainder of the supernatant was assayed for protein following the method of Lowry et al. (1951).

We used the intact rabbit neural retina, rather than the eyecup preparation, which consists of the neural retina, pigment epithelium, and choroid, because it has been reported that the pigment epithelium releases ATP, a possible source of extracellular adenosine (Mitchell, 2001). Thus, use of the intact neural retina allowed us to directly and clearly investigate how light/dark adaptation and the circadian clock regulate intracellular and extracellular adenosine in the neural retina without the presence of the pigment epithelium to complicate interpretation of the findings. We recorded the electroretinogram (ERG) during experiments performed in total darkness to assess the viability of the intact rabbit neural retina preparation. A series of dim white light flashes $(5 \mathrm{sec})$ with intensity ranging from $-10 \log$ Io to $-6 \log$ Io was delivered once every $2 \mathrm{~min}, 1$, 2 , and $3 \mathrm{hr}$ after surgery. The maximum, unattenuated intensity (Io) of full-field white light stimuli from a $100 \mathrm{~W}$ tungsten-halogen lamp was $2.0 \times 10^{3} \mu \mathrm{W} \mathrm{cm}{ }^{-2}$. Calibrated neutral density filters were used to control light intensity. The threshold of the ERG in the day was $-7 \log I$ Io at all time points. At the maximum intensity tested $(-6 \log I 0)$, the amplitude of the b-wave of the ERG, which is primarily generated by bipolar cells (Stockton and Slaughter, 1989), was $43 \pm 2 \mu \mathrm{V}$ after $1 \mathrm{hr}$ and $44 \pm 0 \mu \mathrm{V}$ after $3 \mathrm{hr}$ of superfusion (data are from three retinas from three independent experiments). The kinetics of the b-wave at this light intensity was also similar after 1 and $3 \mathrm{hr}$ of superfusion. That is, the time-to-peak was $203 \pm 26 \mathrm{msec}$ after $1 \mathrm{hr}$ and $175 \pm 28 \mathrm{msec}$ after $3 \mathrm{hr}$, and the half-decay time was $204 \pm 4 \mathrm{msec}$ after $1 \mathrm{hr}$ and $208 \pm 8 \mathrm{msec}$ after $3 \mathrm{hr}(n=3)$. The amplitude of the sPIII, which is generated by Muller glial cells in response to light-induced, photoreceptor-mediated changes in the extracellular $\mathrm{K}^{+}$concentration (Hanitzsch, 1973; Witkovsky et al., 1975), was $168 \pm 19$ and $131 \pm 2 \mu \mathrm{V}$, and the time constant of the sPIII was $1.85 \pm 0.03$ and $1.92 \pm 0.17 \mathrm{sec}$ at the same intensity and time points, respectively (data are from three retinas from three independent experiments). These measurements indicate that the sPIII- and b-waves generated by the intact neural rabbit retina had similar amplitude and kinetics as those typically obtained from the intact rabbit eye (Dong and Hare, 2002) and rabbit eyecup (Dick et al., 1985), and thus that the intact neural retinal preparation is physiologically viable.

Adenosine measurements. Reversed-phase HPLC with fluorescence detection was used to determine the total content of adenosine from retinal homogenates and the amount of endogenous adenosine released into the superfusate from explanted retinas. The liquid chromatograph and detection system consisted of the following: a 515 HPLC pump (Waters, Milford, MA), an automatic refrigerated injector (Agilent 1100 series; Agilent, Waldbronn, Germany), and an adsorbsphere HPLC-silica (C18) column $(250 \times 4.6 \mathrm{~mm} ; 5 \mu \mathrm{m}$ porous silica; Alltech, Deerfield, IL). The fluorescence detector was an Agilent 1100 Series (Agilent Technologies/ Hewlett-Packard, Huntsville, AL). The excitation-emission wavelengths were 230 and $399 \mathrm{~nm}$, respectively. The range was typically $100 \mathrm{lux} / \mathrm{V}$. We used an isocratic mobile phase composed of $0.05 \mathrm{M} \mathrm{NH}_{4} \mathrm{C}_{2} \mathrm{H}_{3} \mathrm{O}_{2}, \mathrm{pH}$ adjusted to 6.0 with acetic acid (Fisher Scientific, Pittsburgh, PA) before addition of $12 \%$ methanol (Fisher Scientific). The solution was filtered with a $0.2 \mu \mathrm{m}$ pore-size membrane and degassed with helium. The mobile phase flow rate was set to $0.7 \mathrm{ml} / \mathrm{min}$, and the column temperature was set to $30^{\circ} \mathrm{C}$ so that the pressure in the column was $\sim 1500 \psi$. Before each experiment, a standard curve was established using various concentrations of $1, N^{6}$-etheno-adenosine ranging from 1 to $500 \mathrm{pg}$ per injection $(30 \mu \mathrm{l})$. The output signal from the fluorescence detector was digitized by a DA-5 BAS analog-to-digital converter (BAS, West Lafayette, IN). Control of the DA-5 and analysis of the chromatograms were performed using BAS software ChromGraph DA-5 control and ChromGraph Report (BAS), respectively. Analyzed peaks were identified by relative retention times compared with those of external standards and were quantified on the basis of their peak areas.

To determine the amount of adenosine present in the Ringer's solution, $500 \mu \mathrm{l}$ of superfusate was processed directly for derivatization. Derivatization consisted in transforming adenosine into $1, N^{6}$-ethenoadenosine, a fluorescent compound. This procedure allowed the use of fluorescence detection, which has a higher sensitivity compared with direct measurement of adenosine using UV detection (Jacobson et al., 1983; Perrett, 1987). Adenosine present in $500 \mu \mathrm{l}$ of superfusate was derivatized in the presence of $0.5 \mathrm{M}$ chloroacetaldehyde and $0.15 \mathrm{M}$ $\mathrm{NH}^{4+}$-acetate, final volume of $650 \mu \mathrm{l}$ and final $\mathrm{pH}$ of 4.5. The solution was then warmed at $65^{\circ} \mathrm{C}$ for $2 \mathrm{hr}$ and cooled down on ice. Control experiments showed that during this procedure, virtually all adenosine (95-100\%) present in the superfusate is derivatized into $1, N^{6}$-ethenoadenosine. Adenosine was then extracted using an agarose-based resin (aminophenylboronate agarose; ProMetic Biosciences, Freeport, UK). Briefly, $500 \mu \mathrm{l}$ of resin and $400 \mu \mathrm{l}$ of $1 \mathrm{M} \mathrm{NH}^{4+}$-acetate, $\mathrm{pH}$ 9.5, were added to each $650 \mu \mathrm{l} \mathrm{sample.} \mathrm{After} 30 \mathrm{~min}$ of rotation at $4^{\circ} \mathrm{C}$ and centrifugation $\left(14,000 \times g\right.$ at $4^{\circ} \mathrm{C}$ for $\left.15 \mathrm{~min}\right)$, the supernatant was removed, and the pellet was washed with $1 \mathrm{ml}$ of cold water. Thereafter, adenosine was resuspended in $650 \mu \mathrm{l} \mathrm{NH}^{4+}$-acetate, $\mathrm{pH} 3.4$, filtered with a $0.2 \mu \mathrm{m}$ pore-size membrane to remove the resin and impurities, and $30 \mu \mathrm{l}$ was injected into the HPLC system. To determine adenosine content, $400 \mu \mathrm{l}$ of water was added to $100 \mu \mathrm{l}$ of retinal homogenate (final volume of 500 $\mu \mathrm{l})$ and then processed exactly as described above for $500 \mu \mathrm{l}$ of superfusate. Because a fraction of etheno-adenosine was usually lost during the process of extraction, the final values were corrected. The correction was 
achieved using an internal standard of known amount (tubercidin). Control experiments showed that the presence of tubercidin and adenosine did not impair the derivatization and extraction of each other, and that an identical fraction of tubercidin and adenosine was lost during the process. Because the ratio of extraction may vary from one vial to another (indeed, more or less resin may be lost during the process), it was calculated for each vial. Typically, the average extraction efficiency was $\sim 50 \%$ (i.e., $50 \%$ of tubercidin and adenosine was lost during the extraction step). The value of adenosine was thus corrected for each run by taking into account the ratio of tubercidin lost (quantity measured at the end/ quantity added at the beginning). The detection threshold of the HPLC system typically was between 1 and $5 \mathrm{pg}$ per run as determined with standard solutions. The lowest values of adenosine measured were above $15 \mathrm{pg}$ per run and thus at least three times the detection threshold.

Identification of adenosine metabolic pathways. To determine whether the source of extracellular adenosine was intracellular or extracellular, we used a number of selective drugs applied alone or in combination. GMP (1 mм) (Rosenberg et al., 2000) and $\alpha \beta$-methylene adenosine diphosphate $(\alpha \beta \mathrm{mADP} ; 50 \mu \mathrm{M})$ (Newman, 2003) were used as selective inhibitors of 5 -ectonucleotidase, and 6-N,N-diethyl-D- $\beta, \gamma$-dibromomethylene-ATP (ARL 67156; $50 \mu \mathrm{M})($ Newman, 2003) was used as a selective inhibitor of 5 '-ectoATPase. Plasma membrane transport of nucleosides occurs by sodium-dependent (nonequilibrative, concentrative) and sodiumindependent (equilibrative) mechanisms, but sodium-dependent, nonequilibrative transporters are limited to specialized cells and have not been found in the mammalian retina (Griffith and Jarvis, 1996; Paes-DeCarvalho, 2002). The sodium-independent, equilibrative transporters are further subdivided as sensitive $\left(\mathrm{IC}_{50}\right.$ in the nanomolar range) or insensitive $\left(\mathrm{IC}_{50}>1 \mu \mathrm{M}\right.$ ) to $N$-nitrobenzylthioinosine (NBTI) (Griffith and Jarvis, 1996). Because both NBTI-sensitive nucleoside transporter subtypes are expressed in brain tissue (Anderson et al., 1999a,b), we used $10 \mu \mathrm{M}$ NBTI to maximally and selectively block equilibrative transport. Proper controls were performed to ensure that every drug we used did not affect the detection of adenosine. Controls consisted in measuring adenosine in a solution containing adenosine alone, the drug alone, or the drug plus adenosine. These solutions were processed for derivatization and extraction. None of the drug tested (GMP, NBTI, $\alpha \beta \mathrm{mADP}$, ARL 67156) interfered with adenosine derivatization and/or detection. However, in the case of GMP, extra peaks were present, as reported by others (Rosenberg et al., 2000). This background was measured in controls and subtracted from the measured values of adenosine. In addition, although GMP dramatically affected the extracellular levels of adenosine, it did not affect intracellular adenosine levels when applied for $30 \mathrm{~min}$ during the day $(n=4)$ (data not shown). These observations are in agreement with the high selectivity of equilibrative nucleoside transporters for adenosine and the membrane-impermeant properties of GMP. NBTI (dissolved in dimethyl sulfoxide; $0.01 \%$ final) and the other drugs (dissolved in superfusion solution) were added directly to the superfusion solution. Control experiments indicated that $0.01 \%$ dimethyl sulfoxide had no effect on the level of adenosine (data not shown). Drugs were applied for a minimum of $15 \mathrm{~min}$.

Unless specified, all compounds were purchased from Sigma-Aldrich (St. Louis, MO).

Statistics. All data are expressed as the mean \pm SEM of $n$ values. The measured values of adenosine were normalized to the protein content of each retina. To test whether adenosine release varies with the time of collection and/or experimental manipulation, statistical analysis was performed with a one-way repeated-measurements ANOVA, followed by Student-Newman-Keuls multiple comparison test, using GraphPad InStat 3.02 (GraphPad Software, San Diego, CA). To compare two groups of independent values, Student's $t$ test was used.

\section{Results}

\section{Measurement of adenosine efflux from the superfused} rabbit retina

Rabbit neural retinas were isolated during the day and superfused $(0.5 \mathrm{ml} / \mathrm{min})$ for up to $3 \mathrm{hr}$ in the dark. Superfusate $(0.5 \mathrm{ml})$ was collected every $10 \mathrm{~min}$, and adenosine was assayed (Fig. 1). Aden-
A

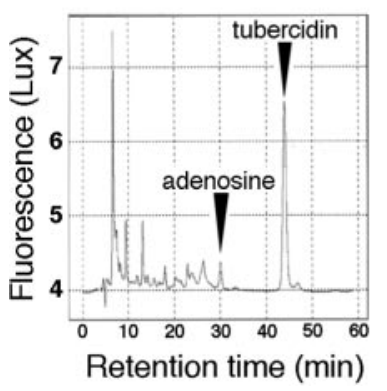

B

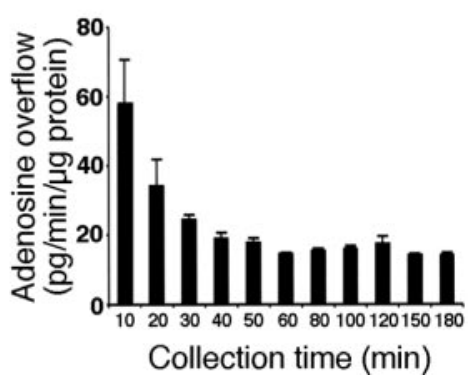

Figure 1. Determination of adenosine overflow from intact superfused rabbit neural retinas. Dark-adapted rabbit neural retinas were isolated during the day and superfused at the rate of $0.5 \mathrm{ml} / \mathrm{min}$ for up to $3 \mathrm{hr}$ in the dark. Superfusate $(0.5 \mathrm{ml})$ was collected every $10 \mathrm{~min}$, and the amount of adenosine in the superfusate was measured. $A$, Typical example of an HPLC chromatogram. The retention time was $30 \mathrm{~min}$ for adenosine and $44 \mathrm{~min}$ for the internal standard tubercidin. Adenosine and tubercidin amount were determined by integration of their peak area. $B$, Time course of adenosine overflow from isolated rabbit neural retinas. Adenosine overflow was high immediately after surgical preparation of the in vitro retinas but stabilized at a lower level after $45 \mathrm{~min}$. Data are from four retinas $\pm \mathrm{SEM}$.

osine was detectable in the superfusate at every time point over the course of the experiment. Although a surge in adenosine overflow was typically observed immediately after surgical isolation of retinas, adenosine overflow from in vitro neural retinas gradually decreased with time and stabilized after $45 \mathrm{~min}$. For this particular reason, in each of the following experiments, the retinas were allowed to recover from surgery for $45 \mathrm{~min}$ in the dark before collecting the superfusate and/or using experimental manipulations.

\section{Effect of time of day and lighting conditions on adenosine release}

To determine whether light affects adenosine release, a background light (white light in the low photopic range; $0.2 \mu \mathrm{W} / \mathrm{cm}^{2}$ ) was applied for $15 \mathrm{~min}$ after a $45 \mathrm{~min}$ period in the dark. Experiments were performed both during the day [Zeitgeber time (ZT) $4-7$, where ZT 0 was dawn] and night (ZT 16-19). Light clearly decreased adenosine overflow at both times of day (Fig. 2A,B). Under these conditions, adenosine overflow recovered to normal dark levels after a 30 min period in the dark. Kinetic analysis of the recovery showed that maximum levels were reached $15 \mathrm{~min}$ after lights were turned off (Fig. 2C). Increasing light intensity resulted in a more pronounced decrease in adenosine release (Fig. $2 D)$. However, at high intensities, adenosine overflow did not recover to its initial level after a $30 \mathrm{~min}$ period of darkness, an effect likely related to the fact that the in vitro neural retinas, which were isolated from the pigmented epithelium, appeared completely bleached from the high intensity illumination. Lower light intensities were also tested. Application of a background light in the scotopic range (intensity of $0.002 \mu \mathrm{W} / \mathrm{cm}^{-2}$ ) for 15 min after a 45 min period in the dark during the day or night did not affect adenosine overflow. In contrast, a background light in the mesopic range (intensity of $0.02 \mu \mathrm{W} / \mathrm{cm}^{-2}$ ) did not have an effect during the day but significantly decreased adenosine overflow at night $(-22 \pm 3 \% ; p<0.01$; paired Student's $t$ test; $n=4)$ (data not shown). These observations establish a threshold light intensity for the modulation of adenosine overflow and confirm that the rabbit retina is more sensitive to light at night than during the day, as reported previously (Brandenburg et al., 1983).

Interestingly, adenosine overflow was higher under dark- 
A

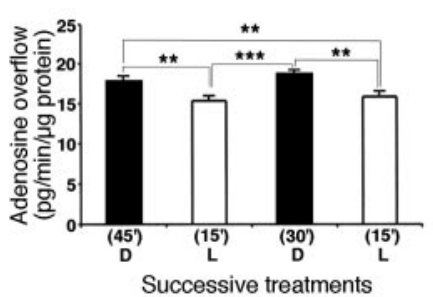

C

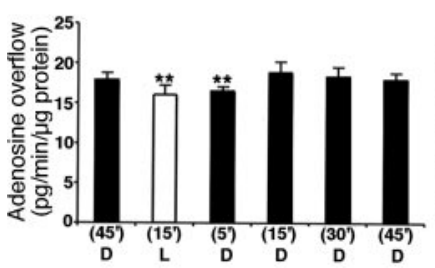

Successive treatments
B

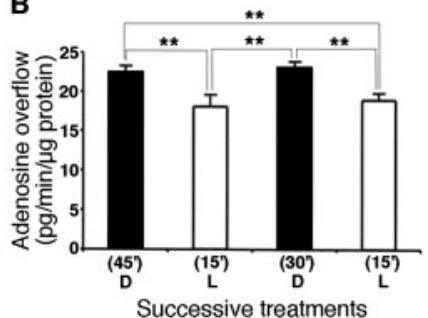

D

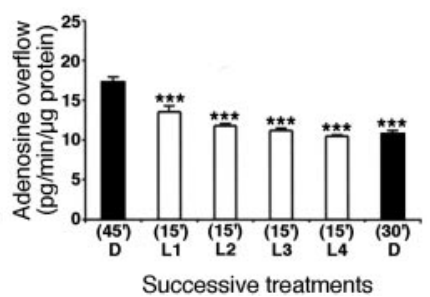

Figure 2. Effect of light illumination and time of day on the extracellular level of adenosine. $A, B$, Dark-adapted rabbit neural retinas were isolated during the day $(A)$ and night $(B)$ and superfused in the dark (D) for $45 \mathrm{~min}$, at the end of which light $\left(\mathrm{L} ; 0.2 \mu \mathrm{W} / \mathrm{cm}^{2}\right)$ and dark conditions alternated as indicated. C, After a $15 \mathrm{~min}$ light exposure at $0.2 \mu \mathrm{W} / \mathrm{cm}^{2}$, adenosine release recovered rapidly to its initial dark levels within $15 \mathrm{~min}$. Note that adenosine levels measured at the end of the initial 45 min period in the dark were higher during the night compared with the day $(A, B) . D$, Brighter intensities of light (ranging from 0.2 to $200 \mu \mathrm{W} / \mathrm{cm}^{2}$ ) applied during the daytime further decreased adenosine overflow. No recovery after a $30 \mathrm{~min}$ period of darkness was observed after a $15 \mathrm{~min}$ application of the most intense light. L1, 0.2 $\mu \mathrm{W} / \mathrm{cm}^{2} ; \mathrm{L} 2,2 \mu \mathrm{W} / \mathrm{cm}^{2} ; \mathrm{L} 3,20 \mu \mathrm{W} / \mathrm{cm}^{2} ; \mathrm{L} 4,200 \mu \mathrm{W} / \mathrm{cm}^{2}$. A fraction of superfusate $(0.5 \mathrm{ml})$ was collected at the end of every period indicated and adenosine assayed. Data are from four to five retinas per condition $\pm S E M ;{ }^{* *} p<0.01,{ }^{* * *} p<0.001$ between the indicated pairs $(A, B)$ or when compared with D $\left(45^{\prime}\right)(C, D)$ (Student-Newman-Keuls multiple comparison test).

adapted conditions during the night compared with the day $(+27 \pm 5 \%$ from five independent experiments; $p<0.001$; Student's $t$ test) (Fig. $2 A, B$ ). These observations suggest that the level of extracellular adenosine is also regulated by a circadian clock (see Fig. 5).

\section{How does darkness increase adenosine overflow?}

To determine how darkness increases adenosine overflow, the effects of darkness were tested in the presence of the adenosine transport blocker NBTI $(10 \mu \mathrm{M})$ and in the presence of NBTI plus the $5^{\prime}$-ectonucleotidase inhibitor GMP (1 mM). Experiments depicted in Figure 3 show that under light-adapted conditions, application of NBTI alone increased adenosine overflow and reversed the light-induced decrease in adenosine overflow, a finding consistent with a flux of adenosine directed from the extracellular space toward the intracellular compartment. Moreover, NBTI did not prevent the dark-induced increase in extracellular adenosine, suggesting that the effects of darkness are mediated primarily by an increase in the extracellular production of adenosine rather than its intracellular formation. Additional application of GMP in the presence of NBTI decreased adenosine levels to an undetectable value, providing additional evidence for an extracellular origin of adenosine. Using the same experimental design, application of NBTI plus $\alpha \beta \mathrm{mADP}(50 \mu \mathrm{M})$, which also selectively blocks $5^{\prime}$-ectonucleotidase, and NBTI plus ARL67156 $(50 \mu \mathrm{M})$, an inhibitor of $5^{\prime}$-ectoATPase, dramatically decreased extracellular adenosine as well (Fig. $3 B$ ). Similar results were observed during the day (Fig. $3 A, B$ ) and night (Fig. 3C,D). However, note that in the presence of NBTI, adenosine levels were increased to a greater extent during the day compared with the

A

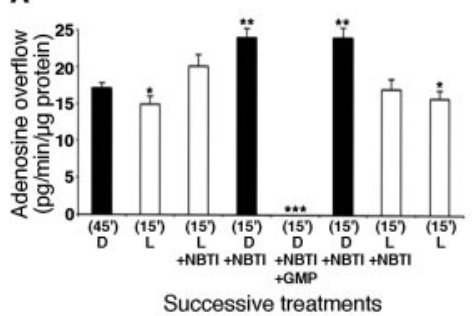

C
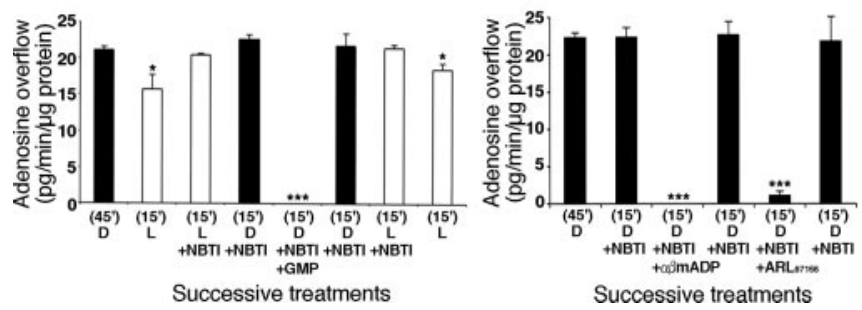

Figure 3. Dark-induced increase in adenosine overflow requires the extracellular production of adenosine from ATP. Isolated neural retinas were superfused in the dark (D) for 45 min after surgery in the day $(A, B)$ and night $(C, D)$. Thereafter, the retinas were superfused in the presence of light (L) for $30 \mathrm{~min}$ ( $15 \mathrm{~min}$ without drug followed by $15 \mathrm{~min}$ with $10 \mu \mathrm{MNBTI})$. The effect of darkness was then tested in the presence of NBTI $(10 \mu \mathrm{M})$ and NBTI plus GMP (1 mM) $(A, C)$, NBTI plus $\alpha \beta \mathrm{mADP}(50 \mu \mathrm{M})$, or NBTI plus ARL67156 (50 $\mu \mathrm{M})(B, D)$. A 15 min period of recovery was allowed between the consecutive applications of NBTI plus $\alpha \beta \mathrm{mADP}$ and NBTI plus ARL67156 $(B, D)$. Although darkness stimulated the accumulation of extracellular adenosine in the presence of NBTI, addition of an inhibitor of the extracellular synthesis of adenosine suppressed the dark-induced increase in adenosine levels. These observations suggest that the dark-induced increase in the extracellular level of adenosine is of extracellular origin. Note that the effects of NBTI were more pronounced during the day than during the night. A fraction of superfusate $(0.5$ $\mathrm{ml}$ ) was collected at the end of every period indicated and adenosine assayed. Data are from four to five individual retinas per condition $\pm \mathrm{SEM} ;{ }^{*} p<0.05,{ }^{* *} p<0.01,{ }^{* * *} p<0.001$ when compared with D (45') (Student-Newman-Keuls multiple comparison test).

night. In the absence of NBTI, application of GMP (1 mM), $\alpha \beta \mathrm{mADP}(50 \mu \mathrm{M})$, or ARL67156 (50 $\mu \mathrm{M})$ alone for $30 \mathrm{~min}$ in the dark dramatically decreased adenosine overflow down to undetectable values both during the day and night (data not shown). Considered together, these observations demonstrate that under dark conditions, extracellular adenosine is primarily produced extracellularly. In addition, the specific effects of ARL67156 further indicate that the primary substrate for adenosine synthesis is likely ATP.

\section{What is the origin of the basal level of adenosine?}

The previous set of experiments suggests that under darkadapted, compared with light-adapted, conditions, extracellular adenosine originates primarily from the extracellular synthesis of adenosine. Using a similar approach, we investigated the origin of the basal levels of adenosine that are observed during the day under light-adapted conditions. As shown previously (Fig. 3), 15 min of light application decreased adenosine overflow compared with the level measured after $45 \mathrm{~min}$ of dark adaptation (Fig. $4 A$ ). Under these conditions, superfusion of NBTI $(10 \mu \mathrm{M})$ increased adenosine overflow, an effect consistent with a flux of adenosine directed toward the intracellular space. Additional superfusion of GMP (1 mM) dramatically decreased adenosine overflow, an effect similar to that observed under dark-adapted conditions and consistent with an extracellular origin of the purine. Application of $\alpha \beta \mathrm{mADP}(50 \mu \mathrm{M})$ and ARL67156 (50 $\mu \mathrm{M})$ under the same conditions provided additional evidence for an extracellular origin of adenosine (Fig. 4B). These observations indicate that the mechanisms regulating the extracellular level of adenosine are 
A

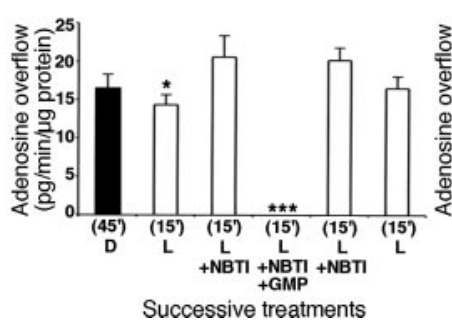

B

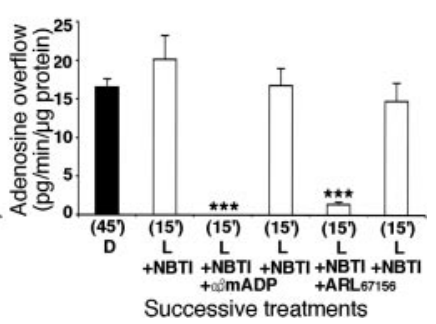

Figure 4. Basal level of extracellular adenosine relies on the extracellular production of adenosine from ATP. Dark-adapted rabbit neural retinas were isolated during the day and superfused in the dark (D) for $45 \mathrm{~min}$, after which light $\left(L ; 0.2 \mu \mathrm{W} / \mathrm{cm}^{2}\right)$ was applied constantly for the remainder of the experiment (the first 15 min without any drug). Thereafter, the effects of NBTI (10 $\mu \mathrm{M})$, NBTI plus GMP (1 mM) (A), NBTI plus $\alpha \beta \operatorname{mADP}(50 \mu \mathrm{M})$, or NBTI plus ARL67156 $(50 \mu \mathrm{m})(B)$ were tested. A 15 min period of recovery was allowed between the consecutive applications of NBTI plus $\alpha \beta$ mADP and NBTI plus ARL67156 ( $B)$. A fraction of superfusate $(0.5$ $\mathrm{ml}$ ) was collected at the end of each period, and adenosine was assayed. Although light stimulation decreased adenosine overflow, superfusion of NBTI increased it, and addition of an inhibitor of the extracellular synthesis of adenosine dramatically decreased it. These observations indicate that the light-evoked decrease in adenosine overflow is mediated by a decrease in the extracellular conversion of ATP into adenosine. Data are from four retinas per condition \pm SEM; ${ }^{*} p<0.05,{ }^{* * *} p<0.001$ when compared with D (45') (Student-Newman-Keuls multiple comparison test).

similar in light- and dark-adapted conditions (i.e., they both rely on the extracellular conversion of ATP into adenosine).

The finding that a basal level of adenosine overflow was still present under light-adapted conditions, even at the highest light intensity used $\left(200 \mu \mathrm{W} / \mathrm{cm}^{2}\right)$ (Fig. $\left.2 D\right)$, but was reduced to an undetectable level after blockade of the extracellular conversion of ATP into adenosine, indicates that ATP is released into the extracellular space under light-adapted conditions. Because light stimulation depolarizes neurons in the ON-pathway (e.g., ONbipolar cells, $\mathrm{ON}$-amacrine cells) and increases the release of synaptic vesicles from them, we investigated whether the basal level of extracellular adenosine originates from ON-pathway neurons by studying the effects of the metabotropic glutamate agonist L-(+)-2-amino-4-phosphonobutyric acid (APB), which selectively blocks all ON light responses (Slaughter and Miller, 1981), on adenosine overflow. Application of APB $(50 \mu \mathrm{M})$ for $30 \mathrm{~min}$ under bright light conditions $\left(200 \mu \mathrm{W} / \mathrm{cm}^{2}\right)$ during the day had no effect on the basal level of extracellular adenosine $(n=4)$ (data not shown), suggesting that the fraction of ATP responsible for the basal level of extracellular adenosine is of glial (nonneuronal) origin (Newman, 2003).

\section{Circadian control of the extracellular level of adenosine}

Because (1) adenosine release in darkness was greater during the night than during the day (Fig. 2), (2) a circadian clock decreases retinal $\mathrm{pH}$ (Dmitriev and Mangel, 2000, 2001) and increases retinal energy metabolism (Dmitriev and Mangel, 2004), and (3) an increase in energy metabolism and/or decrease in $\mathrm{pH}$ increase the level of adenosine in the CNS (Doolette, 1997; Dunwiddie and Masino, 2001), we investigated whether a circadian clock regulates adenosine release in the rabbit retina. Intact neural rabbit retinas were isolated during the subjective day [Circadian time (CT) 5 ; lights off $+17 \mathrm{hr}$ in constant darkness] and subjective night (CT 17; lights off $+29 \mathrm{hr}$ in constant darkness) and were superfused in constant darkness for 45 min after surgery under dim red illumination. At the end of the incubation period, both adenosine overflow and the retinal content of adenosine were assayed. Adenosine overflow displayed a subjective day-night
A

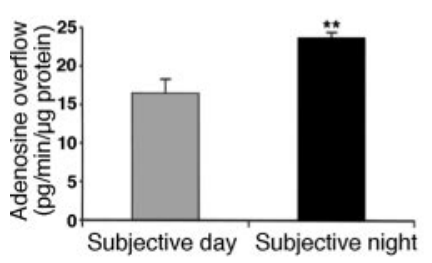

B

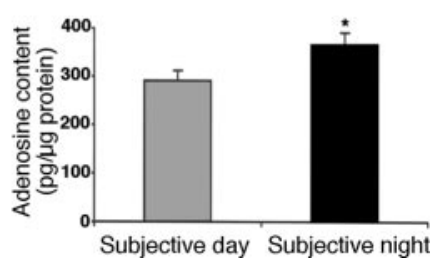

Figure 5. A circadian clock increases adenosine overflow and content in the rabbit retina at night. Neural rabbit retinas were isolated during the subjective day (gray bar) and subjective night (dark bar) and superfused in the dark for $45 \mathrm{~min}$, at the end of which adenosine was assayed in the superfusate $(A)$ and the retina $(B)$ (see Materials and Methods for details). Data are from five retinas per condition $\pm \mathrm{SEM} ;{ }^{*} p<0.05,{ }^{* *} p<0.01$ when compared with subjective day (Student's $t$ test).
A

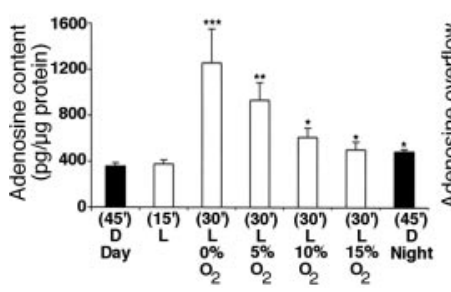

B

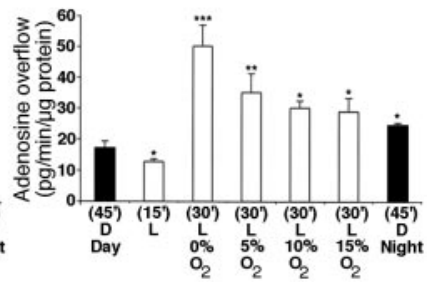

Figure 6. Anoxia and hypoxia increase adenosine content and overflow. Dark-adapted rabbit neural retinas were isolated during the day and superfused in the dark (D) for $45 \mathrm{~min}$, at the end of which light $\left(\mathrm{L} ; 0.2 \mu \mathrm{W} / \mathrm{cm}^{2}\right)$ was applied for the remainder of the experiment. After 15 min in normoxic conditions $\left(5 \% \mathrm{CO}_{2}-95 \% \mathrm{O}_{2}\right)$, hypoxia-anoxia was applied for $30 \mathrm{~min}$, at the end of which adenosine content $(A)$ and the amount of adenosine in the superfusate $(B)$ were assayed. $A$, Effects of different anoxic-hypoxic conditions on retinal adenosine content. After 45 min in darkness, a 15 min light exposure did not significantly change adenosine content $(p>0.5)$. However, a subsequent 30 min period under anoxic conditions $\left(5 \% \mathrm{CO}_{2}-0 \% \mathrm{O}_{2} /\right.$ $90 \% \mathrm{~N}_{2}$ ) led to a dramatic accumulation of intracellular adenosine. Under hypoxic conditions, the effect on adenosine content was greater when the proportion of oxygen was lower (ranging from 5 to 15\%). The amount of adenosine content at night is shown for comparison. A slightly hypoxic $\left(15 \% \mathrm{O}_{2}\right)$ condition increased adenosine content to the same extent as the circadian clock at night. Note that only one anoxic-hypoxic condition was tested per experiment, because the retina was homogenized at the end of the anoxic-hypoxic period. $B$, Effects of different anoxic- hypoxic conditions on the extracellular level of adenosine. Adenosine overflow was measured at the end of each treatment illustrated in $A$. The amount of adenosine measured in the superfusate paralleled the amount of intracellular adenosine. That is, decreasing the proportion of oxygen in the gas mixture dramatically increased adenosine overflow. Data are from four to five retinas per condition $\pm \mathrm{SEM} ;{ }^{*} p<0.05,{ }^{* *} p<0.01,{ }^{* * *} p<0.001$ when compared with $D\left(45^{\prime}\right)$ day (Student-Newman-Keuls multiple comparison test).

difference with $\sim 30 \%$ increase during the subjective night ( $p<$ 0.01 ) (Fig. $5 A$ ). Adenosine content also increased significantly by $\sim 25 \%$ during the subjective night $(p<0.05)$ (Fig. $5 B$ ). These measurements indicate that a circadian clock increases adenosine overflow and content during the subjective night compared with the subjective day. Although light (at the intensity used, $0.2 \mu \mathrm{W} /$ $\mathrm{cm}^{2}$ ) decreased adenosine overflow compared with darkness both during the day and night (Figs. 2-4, 6B), light had no effect on adenosine content during the day (Fig. $6 \mathrm{~A}$ ).

\section{Effect of hypoxic conditions on extracellular and intracellular} levels of adenosine

Because experimental ischemia, anoxia, and hypoxia increase adenosine content in the CNS (Dunwiddie and Masino, 2001; Latini and Pedata, 2001), we studied whether anoxia and hypoxia increase adenosine content and release in the rabbit retina. We investigated whether changing the proportion of oxygen in the gas mixture that bubbles the superfusion solution affected aden- 
osine content and/or overflow in our conditions. Neural rabbit retinas were isolated during the day and were superfused under normoxic conditions $\left(5 \% \mathrm{CO}_{2}-95 \% \mathrm{O}_{2}\right)$ in constant darkness for $45 \mathrm{~min}$ and then for $15 \mathrm{~min}$ in the presence of light $(0.2 \mu \mathrm{W} /$ $\mathrm{cm}^{2}$ ). Thereafter, the light was kept on, and the retinas were superfused for 30 additional min with a superfusion solution of the same composition but bubbled with different gas mixtures. Different concentrations of oxygen were tested, ranging from 0 to $15 \%\left(5 \% \mathrm{CO}_{2}\right.$-balanced with $\left.\mathrm{N}_{2}\right)$. Under anoxic conditions $(0 \%$ $\mathrm{O}_{2}$ ), both adenosine overflow and the retinal content of adenosine were dramatically increased by $\sim 300 \%$ (Fig. 6). Increasing the proportion of oxygen in the mixture decreased the effect of hypoxia down toward initial values (Fig. 6). Interestingly, the effect of a slightly hypoxic $\left(15 \% \mathrm{O}_{2}\right)$ solution on adenosine content and release was similar to that of the circadian clock at night (Fig. 6).

\section{Discussion}

In this study, we monitored endogenous adenosine overflow from intact neural rabbit retina to investigate how it is regulated. We demonstrate for the first time that adenosine in the CNS is modulated by a circadian clock, an endogenous neural process. Moreover, our observations indicate that although both the lighting conditions and a circadian clock modulate the extracellular level of adenosine, light/dark adaptation and the circadian clock likely act through different mechanisms. Specifically, our results indicate that the clock-induced increase in the level of extracellular adenosine at night results primarily from an increase in the level of intracellular adenosine, but that the dark-evoked increase results primarily from an increase in the extracellular conversion of ATP into adenosine. These observations are discussed in more detail below.

Environmental lighting conditions modulate the extracellular synthesis of adenosine

Our findings indicate clearly that the extracellular level of adenosine is controlled directly by the environmental lighting conditions. Indeed, the level of extracellular adenosine was the highest under dark-adapted conditions and decreased when a light background was present. In addition, we found that the suppressive effect of light on extracellular adenosine increased with light intensity. Although it was not possible under our experimental conditions (retina isolated from the epithelium) to observe full recovery of the extracellular adenosine level in the dark after a period of intense light illumination, our observations nonetheless indicate that during bright light illumination, the extracellular level of adenosine is likely the lowest. Overall, our results are in complete agreement with the darkness- and nighttimemimicking effects of exogenous adenosine in the vertebrate retina that have been reported previously (Blazynski and Perez, 1991; Rey and Burnside, 1999; Paes-De-Carvalho, 2002; Sun et al., 2002; Stella et al., 2003).

The source of extracellular adenosine was investigated further using various pharmacological manipulations. First, we found that both basal light and dark levels of extracellular adenosine were increased in the presence of NBTI (Fig. 3). These observations indicate that under both conditions, the flux of adenosine is directed from the extracellular space toward the intracellular compartment, suggesting that extracellular adenosine is formed extracellularly. The effect of NBTI likely resides in the fact that it reduces the transport of adenosine from the extracellular space to the intracellular compartment, leading to extracellular accumulation. This hypothesis was further strengthened by the finding

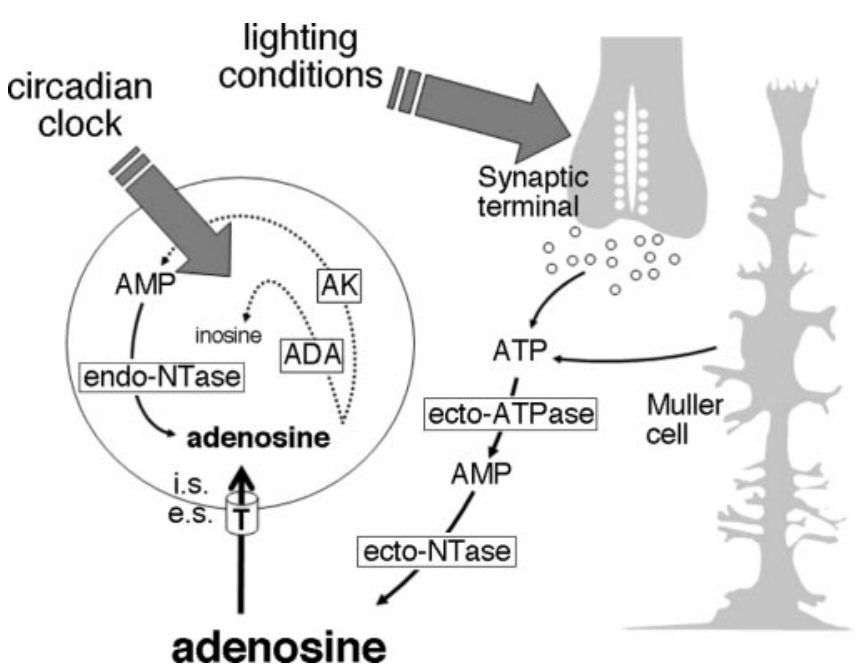

Figure 7. Schematic representation of the dual control of the extracellular level of adenosine by light/dark adaptation and a circadian clock in the mammalian retina. In constant-dark conditions, extracellular adenosine is synthesized from ATP released by synaptic terminals and glial cells via the sequential actions of the ectoATPase and the ectonucleotidase (ectoNTase). Light decreases the level of ATP released from synaptic terminals and thus decreases the level of extracellular adenosine. Although the flux of adenosine is always directed in an inward direction [i.e., the concentration of adenosine is always higher in the extracellular space (e.s.) compared with the intracellular space (i.s.)], the intensity of the flux is lowered at night as a result of the action of a circadian clock. Indeed, the clock increases the intracellular content of adenosine at night, thereby lowering the gradient between the two compartments and the intensity of the inwardly directed flux of adenosine. This clock effect then leads to an accumulation of the purine in the extracellular space at night. The clock either stimulates intracellular adenosine synthesis by the endonucleotidase (endoNTase) at night or inhibits adenosine breakdown by adenosine deaminase (ADA) and adenosine kinase (AK) at night. See Discussion for more details. T, Equilibrative nucleoside transporter.

that application of GMP, $\alpha \beta \mathrm{mADP}$ or ARL67156, alone or in combination with NBTI, decreased adenosine overflow down to undetectable values (Fig. 3). These independent observations are consistent with an extracellular conversion of ATP into adenosine and demonstrate that the extracellular synthesis of adenosine, which is increased by darkness, represents the main source of extracellular adenosine in the mammalian retina (Fig. 7). Possible sources of extracellular ATP include ATP released in synaptic vesicles and/or by Muller glial cells (Dunwiddie and Masino, 2001; Latini and Pedata, 2001; Newman, 2003). Indeed, our observations indicate that a basal level of extracellular adenosine is still present under light-adapted conditions, and that this level is not affected when activity in the ON-pathway is blocked by APB (Slaughter and Miller, 1981), suggesting that part of the extracellular ATP likely originates from glial cells and is not modulated by light and darkness.

Although our measurements indicate that light/dark adaptation modulates the level of extracellular adenosine from synaptically released ATP, our measurements cannot distinguish which neuronal types release the ATP that is converted into adenosine. Moreover, because light stimulation increases the release of synaptic vesicles from some cell types (e.g., ON-bipolar cells) and decreases it from others (e.g., photoreceptors, OFF-bipolar cells), it is possible that light stimulation produces opposite effects on different neuronal types with respect to extracellular adenosine. Our observations therefore indicate the overall effect of light and dark adaptation on extracellular adenosine and may represent an underestimate of the effects of light and dark adaptation on extracellular adenosine with respect to specific neuronal types. 


\section{Circadian control of extracellular adenosine}

How does the circadian clock increase the extracellular level of adenosine during the subjective night? Because our observations indicate that the flux of adenosine is always directed inward under normal physiological conditions, as indicated by the positive effect of NBTI on the extracellular level of adenosine in the day and night (Figs. 3, 4), it can be concluded that the clock does not increase the extracellular level of adenosine during the subjective night by increasing the net flux of adenosine from the intracellular compartment toward the extracellular space. In addition, blockade of the adenosine transporter during the day under darkadapted conditions increased the extracellular level of adenosine to nighttime values, whereas the same treatment barely affected the level of the purine at night (Fig. 3). This observation suggests that the clock likely reduces the inwardly directed flux of adenosine at night rather than increases the extracellular production of adenosine at night.

The nighttime decrease in inward-directed adenosine flux intensity could result from a decrease in transport activity or an increase in the intracellular content of adenosine. Direct measurements of the retinal content of adenosine indicate that the clock increases intracellular adenosine content at night, compared with the day (Fig. 5B). These data are in agreement with an increase in the intracellular concentration of adenosine at night and not with a decrease of transporter activity, because the latter possibility would produce a decrease in retinal adenosine content at night. Together, these data indicate that the circadian clock increases the intracellular content of adenosine at night, which leads to a decrease in the inwardly directed flux of adenosine and an increase in the accumulation of the purine in the extracellular space (Fig. 7).

It is likely that the clock-induced increase in the level of intracellular adenosine at night is a consequence of the circadian clock-induced increase in energy metabolism that has been observed at night in fish and rabbit retinas (Dmitriev and Mangel, 2000, 2001, 2004). Because an increase in energy metabolism likely induces the intracellular accumulation of AMP, a substrate for adenosine, the nighttime clock-induced increase in energy metabolism and neural activity may thus represent the means by which the clock increases the intracellular production of adenosine. An attractive hypothesis is that oxygen consumption may increase at night as a result of the action of the clock, so that a slightly hypoxic condition is generated. Our data agree with this hypothesis, because the clock and slight hypoxia $\left(15 \% \mathrm{O}_{2}\right)$ increased adenosine content and adenosine overflow to a similar extent (Fig. 6). In fact, dark adaptation during the day has been reported to decrease the oxygen tension of the mammalian retina and especially in the synaptic layers and outer nuclear layer, where it reaches $0 \mathrm{mmHg}$ (Linsenmeier, 1986; Ahmed et al., 1993). If the clock produces a slight hypoxic-like state at night, then the resultant increase in adenosine may have a neuroprotective effect (Dunwiddie and Masino, 2001; Latini and Pedata, 2001; Paes-De-Carvalho, 2002) in addition to its neuromodulatory role.

\section{A possible role for the circadian clock in adenosine-mediated homeostatic sleep}

The differential regulation of extracellular adenosine in the retina by a circadian clock and light/dark adaptation (i.e., neural activity) suggests an explanation for how the circadian clock in the brain regulates sleep. It has been proposed that the accumulation of extracellular adenosine during waking acts as an endogenous somnogen and constitutes in part the physiological basis of ho- meostatic sleep need (Porkka-Heiskanen et al., 1997; Shiromani et al., 1998). That is, the longer one is awake and active, the greater the accumulation of extracellular adenosine and the propensity to fall asleep. However, the increase in sleep propensity that occurs as wakefulness-neural activity is prolonged must be integrated with the circadian-mediated regulation of sleep. Moreover, although prolonged wakefulness-neural activity might increase the extracellular accumulation of adenosine by increasing the conversion of extracellular ATP into adenosine, the accumulation of extracellular adenosine will be minimized if the inwardly directed flux of adenosine remains active.

Our present results, which demonstrate that the retinal circadian clock and light/dark adaptation (i.e., neural activity) differentially regulate the extracellular level of adenosine, suggest a mechanism by which the suprachiasmatic nucleus (SCN) clock and homeostatic sleep might interact. Specifically, a circadianevoked increase in intracellular adenosine that occurs concomitantly with a wakefulness-neural activity-evoked increase in extracellular adenosine would augment the accumulation of extracellular adenosine by decreasing its inwardly directed transport. This decrease in the inwardly directed transport of adenosine might occur typically in the late afternoon in diurnal animals, when the effects of the SCN clock and neural activity are high, and toward the end of the night in nocturnal animals, when the effects of the SCN clock and neural activity are high. In roddominated retinas, such as are found in most mammals including humans, neural activity and the effects of the retinal clock are both high at night, leading to the decrease in the inwardly directed transport of adenosine that we have observed. Thus, our results are consistent with an adenosine-mediated two-process model of sleep regulation in which a circadian-induced increase in intracellular adenosine and a wakefulness-neural activityinduced increase in extracellular adenosine act synergistically to enhance the concentration of extracellular adenosine.

In summary, direct measurements of endogenous adenosine overflow and content in the mammalian retina demonstrate for the first time in the CNS that a circadian clock and dark-adaptive processes regulate the neuromodulator adenosine. These results indicate that the dark-evoked increase in the level of extracellular adenosine results primarily from an increase in the extracellular conversion of ATP into adenosine, but that the clock-induced increase at night results primarily from an increase in the level of intracellular adenosine. Moreover, the results suggest that a clock-induced increase in energy metabolism and neural activity at night produces a slight hypoxia-like state that then increases the level of intracellular adenosine.

\section{References}

Ahmed J, Braun RD, Dunn Jr R, Linsenmeier RA (1993) Oxygen distribution in the macaque retina. Invest Ophthalmol Vis Sci 34:516-521.

Anderson CM, Baldwin SA, Young JD, Cass CE, Parkinson FE (1999a) Distribution of mRNA encoding a nitrobenzylthioinosine-insensitive nucleoside transporter (ENT2) in rat brain. Brain Res Mol Brain Res 70:293-297.

Anderson CM, Xiong W, Geiger JD, Young JD, Cass CE, Baldwin SA, Parkinson FE (1999b) Distribution of equilibrative, nitrobenzylthioinosinesensitive nucleoside transporters (ENT1) in brain. J Neurochem 73:867-873.

Barlow R (2001) Circadian and efferent modulation of visual sensitivity. Prog Brain Res 131:487-503.

Blazynski C, Perez MT (1991) Adenosine in vertebrate retina: localization, receptor characterization, and function. Cell Mol Neurobiol 11:463-484.

Brandenburg J, Bobbert AC, Eggelmeijer F (1983) Circadian changes in the response of the rabbit's retina to flashes. Behav Brain Res 7:113-123. 
Cahill GM, Besharse JC (1995) Circadian rhythmicity in vertebrate retinae: regulation by a photoreceptor oscillator. Prog Retin Eye Res 14:267-291.

Dick E, Miller RF, Bloomfield SA (1985) Extracellular $\mathrm{K}^{+}$activity changes related to electroretinogram components. II. Rabbit (E-type) retinas. J Gen Physiol 85:911-931.

Dmitriev AV, Mangel SC (2000) A circadian clock regulates the $\mathrm{pH}$ of the fish retina. J Physiol (Lond) 522:77-82.

Dmitriev AV, Mangel SC (2001) Circadian clock regulation of $\mathrm{pH}$ in the rabbit retina. J Neurosci 21:2897-2902.

Dmitriev AV, Mangel SC (2004) Retinal pH reflects retinal energy metabolism in the day and night. J Neurophysiol 91:2404-2412.

Dong CJ, Hare WA (2002) GABAc feedback pathway modulates the amplitude and kinetics of ERG b-wave in a mammalian retina in vivo. Vision Res 42:1081-1087.

Doolette DJ (1997) Mechanism of adenosine accumulation in the hippocampal slice during energy deprivation. Neurochem Int 30:211-223.

Dunwiddie TV, Masino SA (2001) The role and regulation of adenosine in the central nervous system. Annu Rev Neurosci 24:31-55.

Green CB, Besharse JC (2004) Retinal circadian clocks and control of retinal physiology. J Biol Rhythms 19:91-102.

Griffith DA, Jarvis SM (1996) Nucleoside and nucleobase transport systems of mammalian cells. Biochim Biophys Acta 1286:153-181.

Hanitzsch R (1973) Intraretinal isolation of P3 subcomponents in the isolated rabbit retina after treatment with sodium aspartate. Vision Res 13:2093-2102.

Jacobson MK, Hemingway LM, Farrell TA, Jones CE (1983) Sensitive and selective assay for adenosine using high-pressure liquid chromatography with fluorometry. Am J Physiol 245:H887-H890.

Latini S, Pedata F (2001) Adenosine in the central nervous system: release mechanisms and extracellular concentrations. J Neurochem 79:463-484.

Linsenmeier RA (1986) Effects of light and darkness on oxygen distribution and consumption in the cat retina. J Gen Physiol 88:521-542.

Lowry OH, Rosenbrough NJ, Farr AL, Randall RJ (1951) Protein measurement with the folin phenol reagent. J Biol Chem 193:265-275.

Mangel SC (2001) Circadian clock regulation of neuronal light responses in the vertebrate retina. Prog Brain Res 131:505-518.

Mitchell CH (2001) Release of ATP by a human retinal pigment epithelial cell line: potential autocrine stimulation through subretinal space. J Physiol (Lond) 534:193-202.

Newman EA (2003) Glial cell inhibition of neurons by release of ATP. J Neurosci 23:1659-1666.
Pace-Schott EF, Hobson JA (2002) The neurobiology of sleep: genetics, cellular physiology and subcortical networks. Nat Rev Neurosci 3:591-605.

Paes-De-Carvalho R (2002) Adenosine as a signaling molecule in the retina: biochemical and developmental aspects. An Acad Bras Cienc 74:437-451.

Perrett D (1987) Determination of adenosine ribo- and deoxyribonucleotides as their $1-N^{6}$-etheno derivatives by reversed-phase ion-pair highperformance liquid chromatography. J Chromatogr 386:289-296.

Porkka-Heiskanen T, Strecker RE, Thakkar M, Bjorkum AA, Greene RW, McCarley RW (1997) Adenosine: a mediator of the sleep-inducing effects of prolonged wakefulness. Science 276:1265-1268.

Rey HL, Burnside B (1999) Adenosine stimulates cone photoreceptor myoid elongation via an adenosine A2-like receptor. J Neurochem 72:2345-2355.

Ribelayga C, Wang Y, Mangel SC (2004) A circadian clock in the fish retina regulates dopamine release via activation of melatonin receptors. J Physiol (Lond) 554:467-482.

Rosenberg PA, Li Y, Le M, Zhang Y (2000) Nitric oxide-stimulated increase in extracellular adenosine accumulation in rat forebrain neurons in culture is associated with ATP hydrolysis and inhibition of adenosine kinase activity. J Neurosci 20:6294-6301.

Shiromani PJ, Scammell T, Sherin JE, Saper CB (1998) Hypothalamic regulation of sleep. In: Handbook of behavioral state control: molecular and cellular mechanisms (Lydic R, Baghdoyan HA, eds), pp 311-325. New York: CRC.

Slaughter MM, Miller RF (1981) 2-Amino-4-phosphonobutyric acid: a new pharmacological tool for retina research. Science 211:182-185.

Stella Jr SL, Bryson EJ, Cadetti L, Thoreson WB (2003) Endogenous adenosine reduces glutamatergic output from rods through activation of $\mathrm{A}_{2}$ like adenosine receptors. J Neurophysiol 90:165-174.

Stockton RA, Slaughter MM (1989) B-wave of the electroretinogram. A reflection of ON bipolar cell activity. J Gen Physiol 93:101-122.

Sun X, Barnes S, Baldridge WH (2002) Adenosine inhibits calcium channel currents via A1 receptors on salamander retinal ganglion cells in a minislice preparation. J Neurochem 81:550-556.

Wang Y, Mangel SC (1996) A circadian clock regulates rod and cone input to fish retinal cone horizontal cells. Proc Natl Acad Sci USA 93:4655-4660.

Witkovsky P, Dudek FE, Ripps H (1975) Slow PIII component of the carp electroretinogram. J Gen Physiol 65:119-134. 